\title{
PHOTOEMISSION STUDY \\ OF THE ELECTRONIC STRUCTURE OF SEMICONDUCTING COMPOUNDS
}

\author{
A. FuJimori, J. OKaBAYASHI \\ Department of Complexity Science and Engineering, and Department of Physics \\ University of Tokyo, Bunkyo-ku, Tokyo 113-0033, Japan
}

AND K. MAMIYA

Hiroshima Synchrotron Radiation Center, Hiroshima University

Higashi-Hiroshima 739-8526, Japan

\begin{abstract}
We review how the electronic structure of semiconducting compounds is revealed by photoemission spectroscopy. Angle-resolved photoemission spectroscopy is used to study band dispersions and the photon-energy dependence of spectral line shapes is utilized to study atomic orbital origins of the electronic states, namely, the partial density of states. We focus on recent development in the studies of diluted magnetic semiconductors as well as other magnetic semiconductors, in which interaction between the relatively localized transition-metal $d$ states and the extended $s p$ band states plays a key role in characterizing their unique magnetic, transport, and optical properties.
\end{abstract}

PACS numbers: 79.60.Bm, 71.20.-b, 75.50.Pp

\section{Introduction}

Photoemission spectroscopy is a powerful technique to study the electronic structure of solids [1]. In photoemission experiments, the single-particle spectral function $A(k, \omega)$ multiplied by the Fermi-Dirac distribution function $f(\omega)$, which is the energy and momentum distribution function of electrons in the solid, can be measured through the energy and momentum distribution of photoelectrons emitted from the solid. If photoelectrons are collected over a large solid angle, then the density of states (DOS) of electrons in the solid $N(\omega) f(\omega)$, where

$$
N(\omega)=\int \mathrm{d} k A(k, \omega)
$$

is obtained. In fact, measured spectra are not bare $A(k, \omega)$ or $N(\omega)$ but are modulated by photoionization cross-sections, which are also functions of energy and momentum of electron as well as the polarization of incident light. Photoemission 
measurements give information complementary to that obtained by optical measurements in the sense that photoemission spectra yield the absolute energy positions of occupied states, whereas optical measurements give energy differences between occupied and unoccupied states. Angle-resolved photoemission spectroscopy (ARPES) is a unique experimental technique in that it gives momentum-resolved information about the electronic structure. Note that the absolute positions of unoccupied states can be studied by inverse-photoemission spectroscopy.

Recently, photoemission spectroscopy has made a considerable progress with the use of synchrotron radiation. Because of its energy tunability, synchrotron radiation can be used to study the atomic orbital character of spectral features and also to perform band mapping in three-dimensional materials. The linear polarization of synchrotron radiation can be utilized to obtain unique information in ARPES experiments such as the symmetry of wave functions.

In the following, we review how the electronic structure of semiconducting compounds was revealed by photoemission spectroscopy. Results on the band dispersions and the density of states of typical semiconductors have been extensively studied and are compiled in a previous review article [2]. In this paper, we will focus on the so-called diluted magnetic semiconductors [3] as well as on magnetic semiconductors [4].

\section{Experimental techniques and derived information}

In ARPES experiments of single crystal samples, one measures the distribution of the kinetic energy $E_{\text {kin }}\left(\equiv p^{2} / 2 m\right)$ and the momentum $p$ of photoelectron. From $E_{\text {kin }}$ and $p$, the energy $E_{i}$ and momentum $\hbar k_{i}$ distribution of electrons in the crystalline sample can be obtained using the conservation law of energy and that of momentum parallel to the surface. Assuming the three-step model of the photoemission process (i.e., optical transition in the solid, electron transport to the surface, and escape into the vacuum) and optical transitions into free-electron-like final states, the energy conservation gives

$$
E_{i}+h \nu=\frac{\hbar^{2}\left(k_{i}+G\right)^{2}}{2 m}+V_{0}=\frac{p^{2}}{2 m}+\phi
$$

where $h \nu$ is the photon energy, $G$ is a reciprocal lattice vector, $\phi$ is the work function and $V_{0}$ is the "inner potential" of the sample. Here $E_{i}$ and $V_{0}$ are referenced to the Fermi level $\left(E_{\mathrm{F}}\right)$ and $E_{i}=-E_{\mathrm{B}}$, where $E_{\mathrm{B}}$ is the electron binding energy measured from $E_{\mathrm{F}}$. The momentum conservation is given by

$$
\hbar\left(k_{i}+G\right)_{\|}=(p)_{\|} .
$$

If one fixes the direction of the electron analyzer so as to collect photoelectrons emitted in the direction normal to the surface (normal emission), one can determine band dispersions for $k_{i}$ in the surface normal direction by continuously changing the photon energy. One can also utilize off-normal emission in order to obtain band dispersions over a wider range of the Brillouin zone.

If photoelectrons are collected over a large solid angle or if photoemission experiments are made on a polycrystalline sample, the information about $k_{i}$ is lost and the momentum-integrated DOS is obtained. The measured spectrum is, however, not a real DOS but a DOS modulated by photoionization cross-sections, 
which are strongly dependent on the incident photon energy. Implicitly assuming a tight-binding model for the electronic structure, the measured spectrum is given by the sum of atomic orbital-decomposed DOS (partial DOS) multiplied by the corresponding atomic-orbital photoionization cross-sections. Since the photoionization cross-sections of different atpmic orbitals show different photon energy dependences [5], one can in principle determine the partial DOS using synchrotron radiation with variable photon energy. Most strikingly, if one uses photons near a core-level absorption threshold, the partial DOS of the orbital on the core-excited atom is enhanced due to the so-called resonant photoemission process. This technique is particularly useful to study the transition-metal $d$-derived DOS in transition-metal compounds [6]. In this case, resonant photoemission takes place through interference between the direct emission $d^{n}+h \nu \rightarrow d^{n-1}+\varepsilon_{l}$ and the $p \rightarrow d$ core absorption followed by an Auger-type decay $p^{6} d^{n}+h \nu \rightarrow p^{5} d^{n+1} \rightarrow$ $p^{6} d^{n-1}+\varepsilon_{l}$.

Although indirectly, core-level photoemission spectroscopy also gives useful information about the valence-band electronic structure. When a compound is doped with extra carriers (holes or electrons), the change $\Delta E_{\mathrm{B}}$ in the binding energy of a core-level photoemission peak is given by

$$
\Delta E_{\mathrm{B}}=-\Delta E_{\mathrm{ES}}-\Delta E_{\mathrm{R}}+\Delta \mu,
$$

where $\Delta E_{\mathrm{ES}}$ is the change in the electrostatic potential at the atomic site (which is the sum of the inter-atomic, i.e., Madelung-potential, and intra-atomic contributions), $\Delta E_{\mathrm{R}}$ is the change in the extra-atomic (metallic and dielectric) screening of the core-hole potential, and $\Delta \mu$ is the change in the chemical potential, namely, the Fermi-level shift [7]. $\Delta E_{\mathrm{ESS}}$ should be different between different atoms (especially between anions and cations or between transition-metal and non-transition-metal ions), and $\Delta E_{\mathrm{R}}$ should occur in the opposite directions between photoemission and inverse-photoemission spectra. If various core levels in the photoemission spectra as well as semi-core levels in the inverse-photoemission spectra of a given compound show parallel energy shifts, then we consider that the shifts are due to a shift of the chemical potential. Recently, chemical potential shift has been studied to understand the intriguing changes in the electronic structure of correlated electron systems caused by carrier doping $[7,8]$.

\section{Application to diluted and concentrated magnetic semiconductors}

Energy-band dispersions in semiconductors have been extensively studied by ARPES using synchrotron radiation. In recent years, there has been growing interest in diluted magnetic semiconductors (DMS), in which substituted transition-metal ions add a variety of interesting magnetic and transport properties to the host semiconductors. Mn-substituted III-V compounds including $\mathrm{Ga}_{1-x} \mathrm{Mn}_{x} \mathrm{As}$, whose synthesis became possible recently by molecular beam epitaxy (MBE), have attracted particular attention because of their unique magnetotransport behaviors $[9,10]$. The band structure of GaAs has been obtained by Chang et al. [11] and changes in the band structure induced by Mn substitution in the DMS $\mathrm{Ga}_{1-x} \mathrm{Mn}_{x}$. As has recently been studied using the same technique by Okabayashi et al. [12]. As shown in Fig. 1, the highly dispersive band along the [001] direction 
( $\Delta_{1}$ band) is shifted downward by $0.1-0.2 \mathrm{eV}$, probably due to hybridization with the $\mathrm{Mn} 3 d$ states located at $\approx 4 \mathrm{eV}$ below $E_{\mathrm{F}}$ (see Fig. 2). Furthermore, new states appear to be induced just below $E_{\mathrm{F}}$ by $\mathrm{Mn}$ substitution. These new states are certainly responsible for the novel magnetotransport properties including giant magnetoresistance of this system. Photoemission measurements with high energy resolution and at low temperatures would provide us with more information about the electronic structure near $E_{\mathrm{F}}$ and its relationship to the magnetotransport properties.

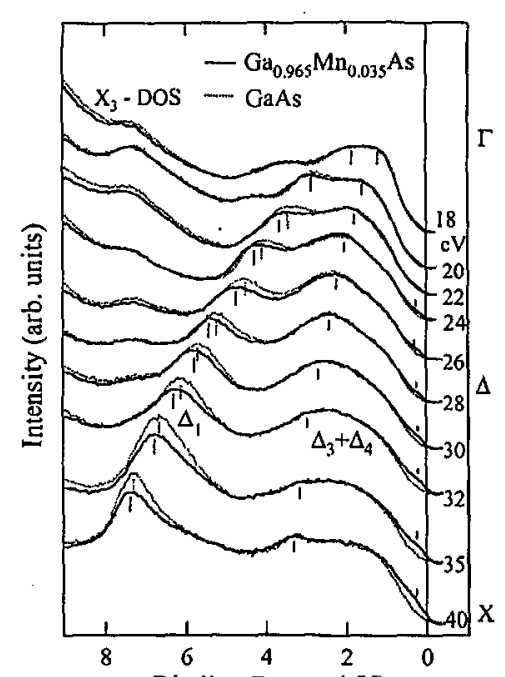

Fig. 1 Binding Energy (eV)

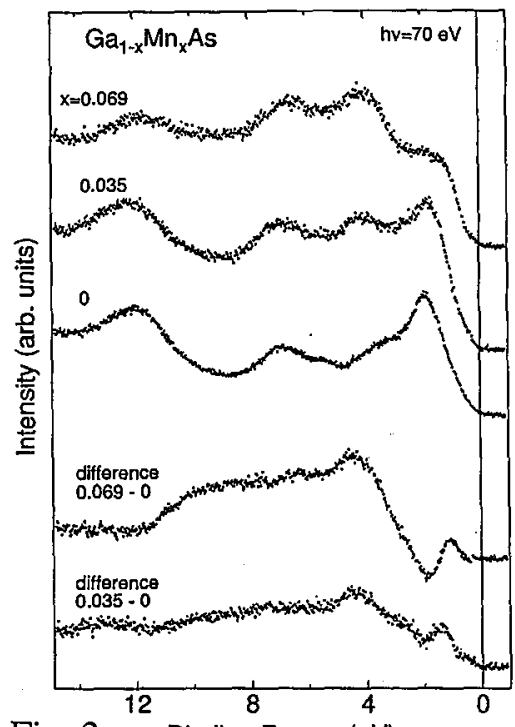

Fig. 2 Binding Energy (eV)

Fig. 1. Angle-resolved photoemission spectra of $\mathrm{GaAs}_{\mathrm{A}}$ and $\mathrm{Ga}_{1-x} \mathrm{Mn}_{x}$ As with $x=$ 0.035 along the [001] direction [12].

Fig. 2. Angle-integrated photoemission spectra of $\mathrm{Ga}_{1-x} \mathrm{Mn}_{x} \mathrm{As}(x=0.035,0.069)$ and GaAs and their difference spectra [13].

Angle-integrated photoemission spectra of GaAs and $\mathrm{Ga}_{1-x} \mathrm{Mn}_{x}$ As are compared in Fig. 2 [13]. Each peak in the spectrum of GaAs corresponds to a van Hove singularity, which gives rise to a high DOS. Changes induced by the Mn substitution are: (i) the peak closest to $E_{\mathrm{F}}$ is broadened and shifted toward lower binding energy $\left(E_{\mathrm{B}}\right)$; (ii) a new peak appears at $E_{\mathrm{B}} \approx 4 \mathrm{eV}$ and the intensity around binding energy $E_{\mathrm{B}} \approx 9 \mathrm{eV}$ shows an increase. Observation (i) probably means that the $\Lambda_{3}$ band along the [111] direction, which yields the high DOS at that binding energy (largely from the $L_{\mathbf{3}}$ point in the Brillouin zone (see p. 365 of Ref. [1])), is shifted upward due to hybridization with the $M n 3 d$ states at $E_{\mathrm{B}} \approx 4 \mathrm{eV}$. As the new peak at $E_{\mathrm{B}} \approx 4 \mathrm{eV}$ can be attributed to the Mn $3 d$ origin, the upward shift of the $\Lambda_{3}$ band and the downward shift of the $\Delta_{1}$ band can be naturally understood as repulsive shifts resulting from the hybridization with the Mn $3 d$ states located between these bands. Note that the Mn $3 d$-derived peak is not seen in the ARPES spectra of Fig. 1 because the Mn $3 d$ photoionization cross-sections is neg- 
ligibly small for such low photon energies. In addition to the hybridization-induced shifts of those bands, a small uniform shift of the entire valence band caused by the chemical potential shift has been inferred from the nearly parallel shifts of the $\mathrm{Ga} 3 d$ and As $3 d$ core levels [10]. A downward chemical potential shift of $\approx 50 \mathrm{meV}$ was found in going from $x=0.035$ to 0.069 , consistent with hole doping.

The Mn $3 d$ partial DOS in DMS was extensively studied using the resonant photoemission technique. Emission from the Mn $3 d$ states is enhanced via interference between the direct photoemission $3 d^{5}+h \nu \rightarrow 3 d^{4}+\varepsilon_{l}$ and the Mn $3 p \rightarrow 3 d$ core absorption followed by an Auger-type decay $3 p^{6} 3 d^{5}+h \nu \rightarrow 3 p^{5} 3 d^{6} \rightarrow 3 p^{6} 3 d^{4}+\varepsilon_{l}$. Figure 3 shows the Mn $3 d$ partial DOS of $\mathrm{Cd}_{1-x} \mathrm{Mn}_{x}$ Te derived from the onand off-resonance spectra [14]. This method has been applied to a number of II-VI-based DMS (see [15]) as well as to $\mathrm{Ga}_{1-x} \mathrm{Mn}_{x} \mathrm{As}$ [13] and the common characteristic "three-peak" structure as shown in Fig. 3 has been obtained. The dominant peak at $\approx 3.5 \mathrm{eV}$ below the valence band maximum (VBM) (which corresponds to the $E_{\mathrm{B}} \approx 4 \mathrm{eV}$ peak in $\mathrm{Ga}_{1-x} \mathrm{Mn}_{x} \mathrm{As}$ ) can be viewed as an "effective" (majority-spin) Mn $3 d$ band whereas the peak near the VBM is due to the Mn $3 d$ character hybridized into the $\mathrm{CdTe}$ valence band. The broad feature centered at $E_{\mathrm{B}} \approx 8 \mathrm{eV}$ (which corresponds to the $E_{\mathrm{B}} \approx 9 \mathrm{eV}$ emission in $\mathrm{Ga}_{1-x} \mathrm{Mn}_{x} \mathrm{As}$ ) is referred to as a multielectron satellite, and reflects electron correlation among the $\mathrm{Mn} 3 d$ electrons. Configuration-interaction (CI) cluster-model analyses of the $\mathrm{Mn}$ $3 d$ partial DOS have led to the conclusion that the satellite is rather due to $\mathrm{Mn}$ $3 d^{4}$ final states whereas the other structures at lower binding energies are due to $3 d^{5} \underline{L}$ final states ( $\underline{L}=$ hole in the valence band), where the Mn $3 d$ hole produced by photoemission is screened by charge transfer from the host valence band to the Mn site [13-15].

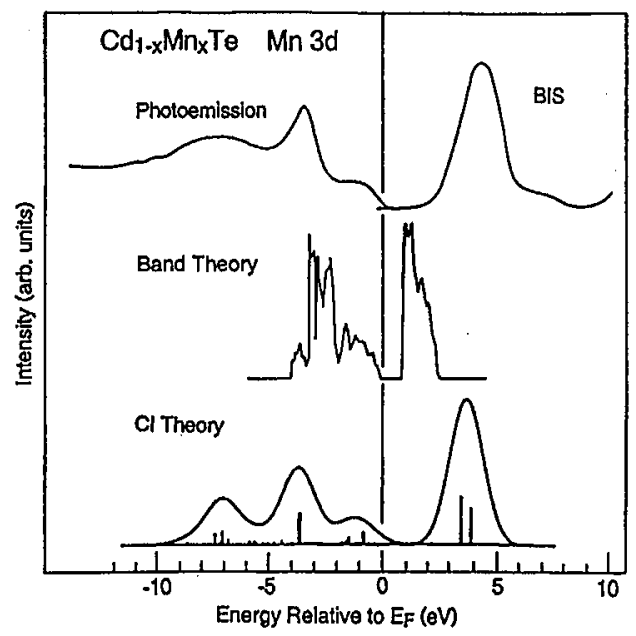

Fig. 3. Mn $3 d$ partial DOS of $\mathrm{Cd}_{1-x} \mathrm{Mn}_{x} \mathrm{Te}(x=0.6)$ deduced by resonant photoemission spectroscopy and inverse-photoemission spectroscopy (BIS) [15] compared with CI cluster-model calculation [14] and band-structure calculation [16]. 
The $x=1$ limit of $\mathrm{Cd}_{1-x} \mathrm{Mn}_{x} \mathrm{Te}$, namely, MnTe with the zinc-blende structure has been synthesized using MBE [17]. The Mn $3 d$ states in MnTe appears to be localized, essentially the same as those in diluted $\mathrm{Cd}_{1-x} \mathrm{Mn}_{x} \mathrm{Te}$. In the chalcopyrite-type $\mathrm{CuAl}_{1-x} \mathrm{Fe}_{x} \mathrm{~S}_{2}$, all the $\mathrm{Al}$ atoms can be replaced by $\mathrm{Fe}$ atoms, leading to $\mathrm{CuFeS}_{2}$ [18]. The magnetic moment of the $\mathrm{Fe}^{3+}$ ions in $\mathrm{CuFeS}_{2}$ is unusually small $\left(3.9 \mu_{\mathrm{B}}\right.$ compared to the free-ion value $5 \mu_{\mathrm{B}}$ of $\left.\mathrm{Fe}^{3+}\right)$ and the Néel temperature is unusually high $\left(T_{\mathrm{N}}=823 \mathrm{~K}\right)$, implying unusually strong $\mathrm{Fe} 3 d-\mathrm{S} 3 p$ hybridization and resulting $\mathrm{Fe} 3 d$ band formation in $\mathrm{CuFeS}_{2}$, in contrast to the Mn $3 d$ states in the zinc-blende MnTe. There has been no ARPES experiment for $\mathrm{CuFeS}_{2}$ whereas the Fe $3 d$ partial DOS has been studied using angle-integrated photoemission [19]. As shown in Fig. 4, the Fe $3 d$ partial DOS derived from the $\mathrm{CuFeS}_{2}-\mathrm{CuAlS}_{2}$ difference spectrum shows qualitatively the same line shape as the Mn $3 d$ partial DOS in the Mn-based DMS and has been analyzed

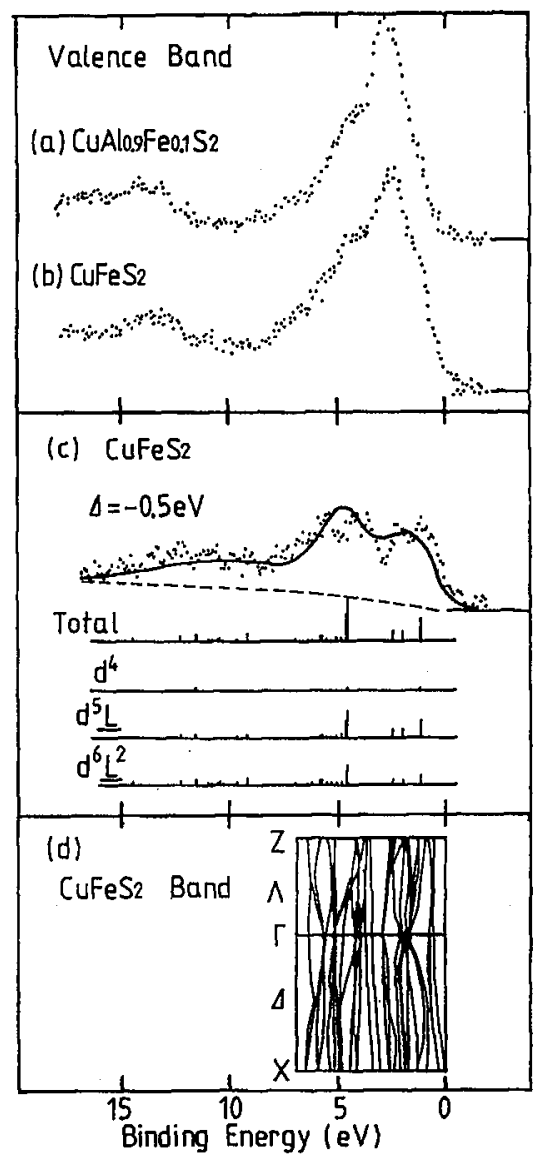

Fig. 4. Photoemission spectra of $\mathrm{CuFeS}_{2}$ (b) and $\mathrm{CuAl}_{0.9} \mathrm{Fe}_{0.1} \mathrm{~S}_{2}$ (a) and their difference spectrum (c) representing the Fe $3 d$ partial DOS [19]. In part (c), the difference spectrum is compared with $\mathrm{CI}$ cluster-model calculation (vertical bars and solid curve). (d) Band structure of $\mathrm{CuFeS}_{2}$. 
with CI cluster-model calculations. The analysis has shown that the $\mathrm{Fe} 3 d$ and $\mathrm{S}$ $3 p$ levels are located very close in energy in the ground state and hence hybridization between them is very strong compared to, e.g., that of the $\mathrm{Mn} 3 d$ and Te $5 p$ levels in $\mathrm{Cd}_{1-x} \mathrm{Mn}_{x} \mathrm{Te}$. This explains the unusual, more band-like behaviors of the $d$ electrons in $\mathrm{CuFeS}_{2}$ compared to the localized $d$-electron system $\mathrm{Cd}_{1-x} \mathrm{Mn}_{x} \mathrm{Te}$.

Transition-metal compounds with the pyrite-type structure, which is a rock-salt arrangement of the $\mathrm{S}_{2}^{2-}$ and divalent metal ions, have been known to show interesting magnetic and transport properties. Among them, $\mathrm{FeS}_{2}$ is a nonmagnetic semiconductor with a band gap of $E_{\mathrm{g}} \approx 0.9 \mathrm{eV}[20]$, and $\mathrm{NiS}_{2}$ is an antiferromagnetic semiconductor with $E_{\mathrm{g}} \approx 0.3 \mathrm{eV}[21]$ and $T_{\mathrm{N}} \approx 40 \mathrm{~K}$ [22]. The band gap of $\mathrm{FeS}_{2}$ is a direct consequence of the filled $t_{2 \mathrm{~g}}$ band of $\mathrm{Fe} 3 d$ and can be well explained by the band theory. Indeed, the photoemission and inverse-photoemission spectra of $\mathrm{FeS}_{2}$ agree well with the band-structure calculation [23] as expected, as shown in Fig. 5 [24]. As for $\mathrm{NiS}_{2}$, on the other hand, because the $e_{\mathrm{g}}$ band of $\mathrm{Ni}$ is partially filled, it is thought to be a Mott insulator, where strong electron-electron interaction opens the band gap in the otherwise metallic system. $\mathrm{MnTe}_{2}$ is also an antiferromagnetic magnetic semiconductor and has been studied using resonant photoemission spectroscopy, revealing the electronic structure of $\mathrm{Mn} 3 d$ to be quite similar to that in $\mathrm{Cd}_{1-x} \mathrm{Mn}_{x} \mathrm{Te}$ [25]. The photoemission spectra of $\mathrm{NiS}_{2}$ show a

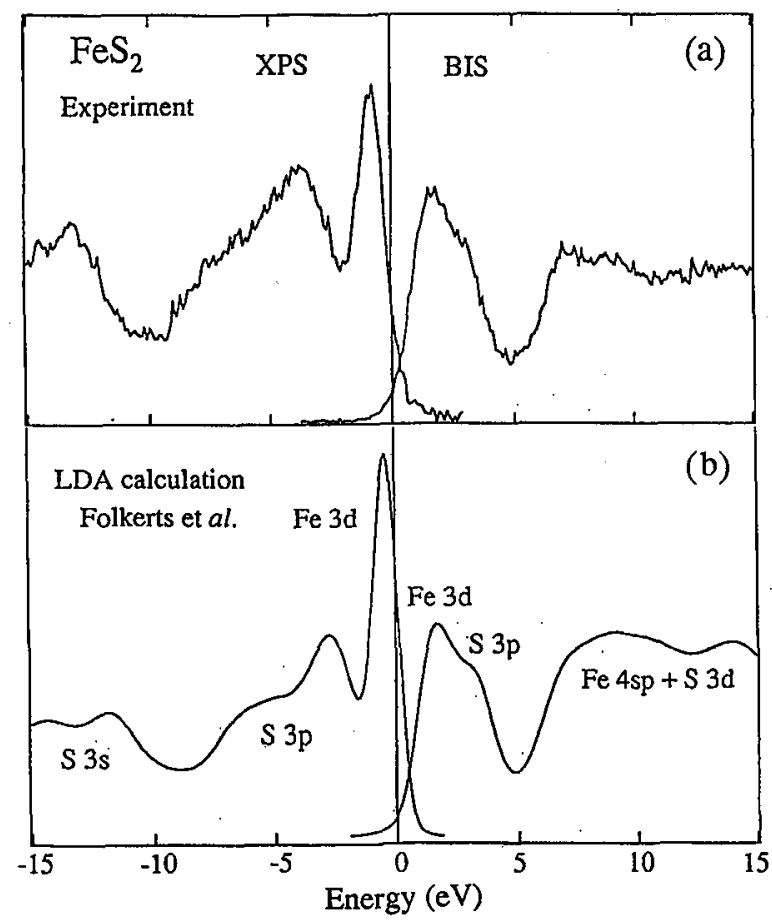

Fig. 5. Photoemission and inverse-photoemission (BIS) spectra of $\mathrm{FeS}_{2}$ (a) [24] compared with the first-principles band-structure calculation (b) [23]. 


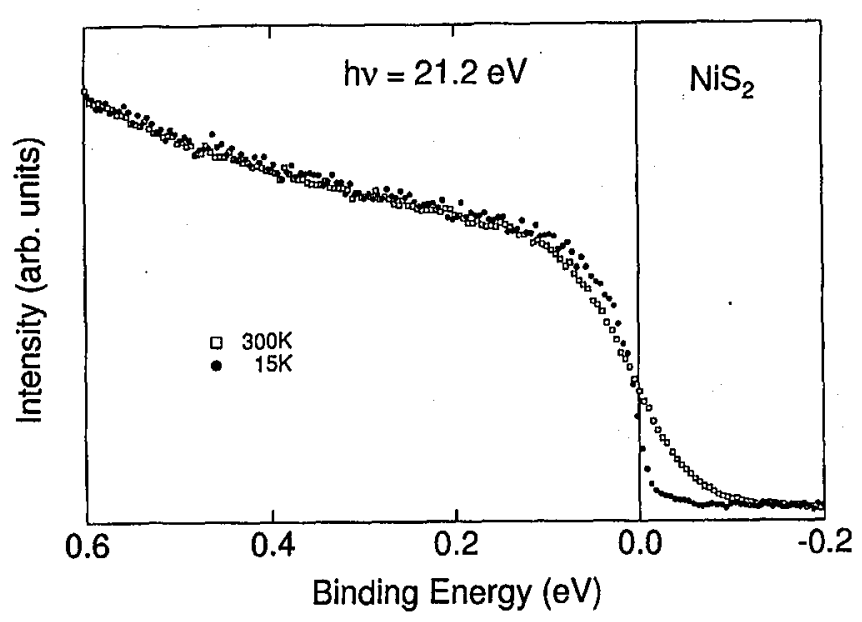

Fig. 6. Photoemission spectra of $\mathrm{NiS}_{2}$ near the Fermi level [27].

satellite [26], and thus differ from those predicted by band-structure calculations as in the case of the Mn-doped DMS.

High-resolution photoemission spectra in the vicinity of $E_{\mathrm{F}}$, especially their temperature dependence, give useful information about the transport mechanism in semiconducting compounds. As for $\mathrm{NiS}_{2}$, the gap is not observed at low temperature in the photoemission spectra as shown in Fig. 6 probably because it shows $p$-type conduction and the Fermi level is located near the VBM [27]. The spectra at room temperature also do not show any visible gap in spite of the gap as large as $300 \mathrm{meV}$ found by the optical study and the $\approx 70 \mathrm{meV}$ activation energy found from the transport study above $170 \mathrm{~K}$. The absence of the gap in the photoemission spectra and hence the proximity of the Fermi level to the VBM at high temperatures implies that the activation energy for the hole carrier number is negligibly small and that a large number of hole carriers are thermally activated. Therefore, in order to explain the activation-type transport in $\mathrm{NiS}_{2}$, the mobility should be activation-type and have an activation energy as large as $\approx 70 \mathrm{meV}$. Indeed, a small mobility $<0.5 \mathrm{~cm}^{2} /(\mathrm{V} \mathrm{s})$ has been deduced from the Hall coefficient measurement, consistent with small polaron hopping.

\section{Acknowledgments}

The author would like to thank T. Mizokawa, A. Kimura, O. Rader, T. Hayashi, M. Tanaka, S. Suga, K. Sato, M. Fujisawa, M. Taniguchi, L. Ley, K. Kaznacheyev, T. Kanomata, D.D. Sarma, and T. Miyadai for fruitful collaboration. This work is partly supported by a Grant-in Aid for Scientific Research on the Priority Area "Spin Controlled Semiconductor Nanostructures" from the Ministry of Education, Science, Sports, and Culture of Japan.

\section{References}

[1] S. Hüfner, Photoelectron Spectroscopy, Springer-Verlag, Berlin 1995.

[2] L. Ley, M. Cardona, in: Photoemission in Solids, Vol. 2, Springer-Verlag, Berlin 1979, p. 11. 
[3] J.K. Furdyna, J. Appl. Phys. 64, R29 (1988).

[4] J.A. Wilson, Adv. Phys. 21, 143 (1972); K. Sato, Prog. Cryst. Growth Charact. 11, 109 (1985).

[5] J.J. Yeh, I. Lindau, At. Data Nucl. Data Tables 32, 1 (1985).

[6] L.C. Davis, J. Appl. Phys. 59, R25 (1986).

[7] A. Fujimori, A. Ino, T. Mizokawa, T. Tsujioka, T. Yoshida, M. Satake, K. Okazaki, H. Eisaki, S. Uchida, K. Kishio, Y. Taguchi, T. Katsufuji, Y. Tokura, Y. Yasui, T. Nishikawa, M. Sato, J. Electron Spectrosc. Relat. Phenom. 92, 59 (1998).

[8] A. Ino, T. Mizokawa, A. Fujimori, K. Tamasaku, S. Uchida, T. Kimura, T. Sasagawa, K. Kishio, Phys. Rev. Lett. 79, 2101 (1997).

[9] H. Ohno, H. Munekata, T. Penny, S. von Molnar, A. Semuller, L.L. Chang, Phys. Rev. Lett. 68, 2664 (1992).

[10] T. Hayashi, M. Tanaka, T. Nishinaga, H. Shimada, J. Appl. Phys. 81, 4865 (1997).

[11] T.C. Chang, J.A. Knapp, M. Aono, D.E. Eastman, Phys. Rev. B 21, 3513 (1980).

[12] J. Okabayashi, A. Fujimori, A. Kimura, O. Rader, T. Hayashi, M. Tanaka, submitted for publication.

[13] J. Okabayashi, A. Kimura, T. Mizokawa, A. Fujimori, T. Hayashi, M. Tanaka, Phys. Rev. B 59, R2486 (1999).

[14] L. Ley, M. Taniguchi, J. Ghijsen, R.L. Johnson, A. Fujimori, Phys. Rev. B 35, 2839 (1987).

[15] A. Fujimori, T. Mizokawa, in: II-VI Semiconductor Compounds, Ed. M. Jain, World Scientific, Singapore 1993, p. 103.

[16] S.-H. Wei, A. Zunger, Phys, Rev. B 35, 2340 (1987).

[17] T. Ando, K. Takahashi, T. Okuda, M. Umehara, Phys. Rev. B 46, 12289 (1992).

[18] T. Teranishi, K. Sato, K. Kondo, J. Phys. Soc. Jpn. 36, 1618 (1974).

[19] M. Fujisawa, S. Suga, T. Mizokawa, A. Fujimori, K. Sato, Phys. Rev. B 49, 7155 (1994).

[20] W.W. Kou, M.S. Seehra, Phys. Rev. B 18, 7062 (1978).

[21] R.L. Kautz, M.S. Dresselhaus, D. Adler, A. Linz, Phys. Rev. B 6, 2078 (1972).

[22] T. Miyadai, K. Takizawa, H. Nagata, H. Ito, S. Miyahara, K. Hirakawa, J. Phys. Soc. Jpn. 39, 63 (1975).

[23] W. Folkerts, G.A. Sawatzky, C. Haas, R.A. de Groot, F.U. Hillebrecht, J. Phys. $C$ 20, 4135 (1987).

[24] K. Mamiya, S. Suga, A. Fujimori, unpublished data quoted by M. Imada, A. Fujimori, Y. Tokura [Rev. Mod. Phys. 70, 1039 (1998)].

[25] K. Kaznacheyev, T. Muro, T. Matsushita, T. Iwasaki, Y. Kuwata, H. Harada, S. Suga, H. Ishii, T. Miyahara, T. Mizokawa, A. Fujimori, T. Harada, T. Kanomata, Phys. Rev. B 58, 13491 (1998).

[26] A. Fujimori, K. Mamiya, T. Mizokawa, T. Sekiguchi, T. Miyadai, H. Takahashi, N. Môri, S. Suga, Phys. Rev. B 54, 16329 (1996).

[27] K. Mamiya, T. Mizokawa, A. Fujimori, T. Miyadai, H. Takahashi, N. Môri, S. Suga, N. Chandrasekharan, S.R. Krishnakumar, D.D. Sarma, Phys. Rev. B 58, 9611 (1998). 